Revista de Filosofía

ISSN: 0034-8244

http://dx.doi.org/10.5209/resf.63051

\title{
La teoría husserliana del tiempo en los textos tempranos (1893-1917)
}

\author{
Verónica Kretschel ${ }^{1}$
}

Recibido: 21 de enero de 2019 / Aceptado: 16 de enero de 2020

Resumen. Las Lecciones de fenomenología de la conciencia interna del tiempo han sido caracterizadas tanto por su enigmática belleza textual como por su dificultades e inconsistencias. El objetivo de esta comunicación es intentar establecer algunos puntos centrales de la teoría del tiempo husserliana en sus textos tempranos, conducido por el texto de las Lecciones y acompañado por la totalidad de escritos del período y las discusiones más relevantes que se han hecho al respecto.

Palabras Clave: fenomenología; Husserl; conciencia del tiempo.

\section{[en] The Husserl's theory of time in early texts (1893-1917)}

\begin{abstract}
The Lectures on Internal Time Consciousness from the Year 1905 were characterised both by its enigmatic beauty and its difficulty and inconsistency. The aim of this paper is to try to establish some of the central points of the Husserl's theory of time in his early writings, guided by the Lectures but with the support of all texts from the period and the most relevant discussions on the matter.
\end{abstract}

Keywords: phenomenology; Husserl; time-consciousness.

Sumario: 1. Introducción; 2. El esquema aprehensión-contenido de aprehensión; 3. Aprehensiones de tiempo y presentificaciones; 4. Problemas del esquema; 5. La conciencia absoluta; 6. Los niveles de la temporalidad; 6.1. Las críticas de Zahavi a la "interpretación standard"; 6.2. Brough y el problema de las vivencias; 7. Conclusión; 8.Referencias bibliográficas.

Cómo citar: Kretschel, V. (2020): "La teoría husserliana del tiempo en los textos tempranos (18931917)", en Revista de Filosofia 45 (2), 227-245. 


\section{Introducción}

Ya se ha explicado por qué las Lecciones de fenomenología de la conciencia interna del tiempo no se pueden leer como una obra que presenta una teoría del tiempo consolidada. La variedad de temas que es abordada en el texto y la cantidad de problemas a los que se pretende responder complican la posibilidad de pensarlas como una obra integral (Lohmar 2010, p. 117). A su vez, las modificaciones terminológicas puestas en juego, las contradicciones patentes en asunciones esenciales, los cambios en el eje de la argumentación, son todos obstáculos frecuentes para quien encara la tarea de comprender este libro (Abella 2005). Gran parte de estas dificultades pueden, con todo, ser salvadas cuando se tiene en cuenta la historia de edición y publicación de las Lecciones. (Ingarden 1962). Así, la posibilidad de conocer la datación de cada parágrafo resulta una herramienta indispensable para organizar su estudio. En este marco, nuestro objetivo aquí es trazar un recorrido por las Lecciones, con el acompañamiento de la totalidad de los textos sobre el tema en el período indicado, buscando establecer algunos puntos centrales para intentar caracterizar la teoría temprana del tiempo en Husserl y poder posicionarnos respecto a las interpretaciones más importantes de la cuestión.

Para llevar a cabo nuestra tarea, comenzaremos con el análisis del primer marco conceptual desde el cual es encarado el fenómeno de la temporalidad; i.e.: el esquema aprehensión-contenido de aprehensión (das Schema Auffasssung-auffgefasstem Inhalt). Seguiremos con las dificultades que ocasiona el esquema y las posibilidades que se plantean respecto a un rechazo o restricción de este modelo y el surgimiento de una instancia independiente, la conciencia absoluta constituyente del tiempo como fundamento último de la temporalidad. Para finalizar con una descripción de esta instancia y los problemas que también ella acarrea, en relación, sobre todo, con las dificultades para establecer el status de las distintas dimensiones de la temporalidad a las que Husserl hace referencia.

\section{El esquema aprehensión-contenido de aprehensión}

El comienzo de los estudios sobre el tiempo está orientado a establecer las condiciones de posibilidad de la percepción de un objeto que dura. Es decir, pretende determinar cómo se conforma "la constitución originaria de objetos temporales, tratando de comprender analíticamente la conciencia del tiempo que se da en ellos" (Hua X, p. 30/p. 52). ${ }^{2}$ En esta etapa de la investigación el modo de pensar la constitución de los objetos temporales coincide con el modelo de constitución de las Investigaciones lógicas; i. e.: la teoría de la representación (Represäntationstheorie) o teoría esquemática. ${ }^{3}$ Se entiende que la constitución de los actos de la conciencia se da en

2 Citaremos las obras de Husserl siguiendo la citación canónica de las obras completas. En el caso de las Lecciones de fenomenología de la conciencia inmanente del tiempo, recogemos la traducción al castellano de Agustín Serrano de Haro e indicamos la paginación respectiva seguida de una barra después del original. Lo mismo para la traducción de Investigaciones Lógicas, a cargo de José Gaos y Manuel García Morente.

3 Brough señala, con todo, que en algunos de los textos más tempranos de Hua X (1901) Husserl sostiene como teoría de la constitución una teoría de la imagen. Esto se observa en el modo de caracterizar la memoria como un acto que requiere la mediación de una imagen presente para establecer el nexo entre el acto de recordar y el objeto recordado. Esta teoría es fuertemente criticada a partir de 1905, lo que implica que en este grupo de textos nos encontramos sobre todo con las objeciones que se le dirigen (Brough 1991, p. XLII) 
términos del esquema aprehensión-contenido de aprehensión. Según este esquema, un contenido sensible inmanente (un componente real de la conciencia que no es él mismo un acto), desprovisto de toda referencia, al ser aprehendido interpretativamente establece una relación intencional con algo objetivo. El acto se concibe, pues, como la combinación de dos componentes: un contenido no-intencional y una aprehensión intencional. Una percepción, por tomar el ejemplo paradigmático, se constituye, por tanto, cuando una aprehensión interpreta un contenido sensible y da lugar a la aparición (Erscheinung) en carne y hueso (leibhaft) de un objeto intencional.

Brough señala que la clave del esquema radica en dos tesis conectadas entre sí (Brough 1977, p. 86). La primera sostiene que los contenidos inmanentes son neutrales con respecto a toda referencia externa. En consonancia con esta, la segunda tesis afirma que la referencia intencional y el rol que el contenido pueda asumir dependen enteramente de la aprehensión que tenga lugar. Por tanto, la aprehensión determina si el contenido es interpretado de tal o cual forma, constituyendo tal o cual referencia objetiva. En ese sentido, afirma Husserl que "la objetividad se constituye no justamente en los contenidos primarios sino en los caracteres de aprehensión" (Hua X, p. 8/p. 30). Esto explica por qué el mismo contenido puede ser aprehendido de modo diferente, remitiendo a distintos objetos. A su vez, apunta a una cuestión central a la hora de caracterizar los distintos aspectos de la relación intencional. Cuando nos referimos a un contenido sensorial, no estamos mentando con ello un aspecto del objeto. La diferencia es patente: mientras que el contenido sensible inmanente es vivido (erlebt), el color del objeto es percibido (Hua XIX/I, pp. 358359/p. 477; Hua X, pp. 6-7/p. 28).

La teoría husserliana toma de la psicología la noción de vivencia. Asume que las vivencias son "los acontecimientos reales (...) que constituyen la unidad real de la conciencia" (Hua XIX/I, p. 357/p. p. 476) y, cuando el psicólogo añade "del individuo psíquico correspondiente" (Hua XIX/I, p. 357/p. 476), Husserl pone entre paréntesis toda conexión empírica y limita el sentido de vivencia a un dominio de pureza fenomenológico. Las vivencias en sentido fenomenológico son, entonces, todos los actos, emociones, voliciones y sus componentes o momentos (es decir: contenidos y aprehensiones de contenido). Todo esto es tomado como contenido real de la conciencia y, en la medida en que forma parte de la vida de la conciencia, es vivido. Se establece, entonces, una diferencia fundamental en el modo de darse de lo inmanente y de lo trascendente:

El fenómeno de la cosa (la vivencia) no es la cosa aparente, la cosa que se halla frente a nosotros supuestamente en su propio ser. Como pertenecientes a la conexión de la conciencia vivimos los fenómenos; como pertenecientes al mundo fenoménico, se nos ofrecen aparentes las cosas. Los fenómenos mismos no aparecen; son vividos. (Hua XIX/I, pp. 359-360/p. 478)

Entonces, mientras que el fenómeno es vivido, el objeto es percibido. Según esto, si queremos comprender cómo se conforma la percepción de un objeto temporal deberemos indagar en la multiplicidad de fenómenos que implica. En las Lecciones Husserl ilustra esta idea del siguiente modo:

Dirijamos la mirada a un trozo de tiza; cerramos y abrimos los ojos. Tenemos entonces dos percepciones, y decimos en consonancia que vemos la tiza dos veces. Tenemos, pues, 
contenidos separados en el tiempo, e intuimos también una disociación fenomenológica temporal, una separación, pero en el objeto no hay separación ninguna, es siempre el mismo: en el objeto, duración; en el fenómeno cambio. (Hua X, p. 8/ p. 30; la cursiva es propia)

Para que podamos percibir algo trascendente, eso trascendente se tiene que manifestar. En ese sentido, nos relacionamos con lo trascendente a través de sus apariciones fenoménicas. Pero: ¿qué pasa si la continuidad de un objeto nos es dada a través de manifestaciones discontinuas? ¿Cómo es posible que ocurran modificaciones a nivel fenoménico que no se sigan de cambios en la objetividad? O, mejor, ¿cómo sabemos de la duración del objeto, si ese objeto se nos manifiesta a través de múltiples apariciones cambiantes? Evidentemente debe existir en la conciencia el mecanismo capaz de posibilitar la unidad del objeto que dura.

Cuando percibimos un objeto temporal, sólo una de sus caras está presente efectivamente. Sin embargo, las caras "ausentes" del objeto están presentes también en cierta medida. Una de las objeciones centrales que le dirige Husserl a Brentano en las Lecciones es el hecho de no considerar la duración de los actos y sólo atribuir carácter temporal a las sensaciones. Frente a esto, la teoría husserliana procura describir la percepción de un objeto temporal, atribuyendo la constitución del tiempo a las aprehensiones implicadas. Desde esta perspectiva, la percepción es tomada como un acto que dura, como un proceso que está conformado por múltiples aprehensiones. Este proceso tiene un comienzo que coincide con la primera aparición del objeto. Un contenido sensible es aprehendido como un aspecto de un objeto trascendente. Pero la duración del objeto coincide con una modificación permanente. Un nuevo contenido sucede al anterior y una nueva aprehensión mantiene la percepción del mismo objeto. Cada contenido que es reemplazado se modifica y se mantiene asociado al presente, siempre que sean aprehendidos como partes del mismo objeto percibido. Es decir que, mientras la aprehensión mantenga la identidad objetiva, el presente se distiende en dirección a los contenidos de aprehensión recién sidos. A su vez, también se produce una ampliación del presente hacia el futuro, en la medida en que nada haga pensar que el objeto intencionado saldrá fuera de nuestro campo perceptivo.

Se sigue de esto que el acto de percepción posee una intencionalidad triple (Hua X, p. 39/ p. 61). Este carácter de la percepción temporal puede deslindarse en las aprehensiones que lo componen. Por un lado, una aprehensión dirigida al contenido efectivamente presente que llamamos fase actual, en la medida en que coincide con la aparición presente del objeto. Por otro lado, aprehensiones que apuntan a los contenidos pasados (recuerdo primario) y aprehensiones que interpretan los contenidos por venir (expectativa primaria). Estas aprehensiones conforman un presente ampliado que permite dar cuenta de la percepción como un acto que tiene una duración y que, a su vez, intenciona un objeto que dura. Esto supone una doble noción de presente (Hua X, p. 40/ p. 61). Hay un presente puntual que se corresponde con la fase actual de la percepción y cuyo correlato trascendente es el ahora como límite. Pero, también, hay un presente distendido, que es el presente percibido y que se constituye a partir de la estructura triple de la conciencia. Y en este punto cabe destacar que, si bien Husserl mantendrá esta idea de pensar al presente en su distensión, tanto los recursos conceptuales, como las elecciones terminológicas se irán modificando con el desarrollo de la teoría. En principio lo que queda firme es 
esta estructura triple de la intencionalidad sobre la cual se articula la duración de la percepción.

Ahora bien, a la pregunta por la posibilidad de la identidad en el objeto, pese a la modificación en el fenómeno, debemos responder que se funda en la permanencia de la aprehensión que interpreta los contenidos. En la medida en que los contenidos de aprehensión son neutrales, toda referencia objetiva está subordinada al componente aprehensivo. En este sentido es que decimos que, mientras la aprehensión siga estableciendo la misma referencia intencional, apuntando al mismo objeto, se anula el problema respecto a la discontinuidad de las manifestaciones (Hua X, p. 215), dado que en términos del esquema aprehensión-contenido de aprehensión el único proceso sintético con el que se cuenta es la aprehensión, entendida como interpretación de la sensación. En desarrollos subsiguientes la fenomenología del tiempo tomará como objeto los demás aspectos de la relación intencional y el esquema en cuestión se mostrará deficitario a la hora de dar cuenta de la constitución de la conciencia temporal de modo acabado.

\section{Aprehensiones de tiempo y presentificaciones}

El esquema es utilizado también tempranamente para describir no sólo la percepción, sino también los distintos tipos de presentificaciones (Vergegenwärtigungen). En este caso, la posibilidad de diferenciar las clases de actos está en relación con el tipo de contenido implicado. Mientras que el contenido de una percepción es una sensación, el contenido de la fantasía, la rememoración y la expectativa es un fantasma. Las presentificaciones no son presentaciones originales de algo efectivamente presente. No obstante, pueden ser intuitivas y, en ese punto, poseen un componente sensorial. Si ese componente fuera la sensación original, entonces sería imposible distinguir la percepción de las presentificaciones. Por tanto, se les atribuye un contenido modificado, al cual se denomina fantasma (Hua X, p. 102/ p. 123). Los fantasmas son contenidos sensibles que se distinguen de las sensaciones originales porque no detentan por sí mismos auténtica realidad; son la "contrafigura de las sensaciones" (Hua X, p. 103/ p. 124).

La pregunta que se impone, entonces, es cómo se aplica este modelo de constitución a las aprehensiones de tiempo. Por tomar un ejemplo: ¿el recuerdo primario es también el resultado de la aprehensión de un fantasma? La respuesta es negativa:

Quien asume una diferencia esencial entre sensaciones y fantasmas no puede naturalmente pretender que los contenidos de aprehensión relativos a las fases temporales recién pasadas sean fantasmas, dado que ellos desembocan de continuo en los contenidos de aprehensión del momento ahora. (Hua X, p. 47/ p. 69)

En la medida en que el recuerdo primario constituye el presente ampliado junto con la percepción ahora y la expectativa primaria, si fuera el resultado de la aprehensión de un fantasma la misma noción de presente perdería su carácter originario y la teoría husserliana sucumbiría, rápidamente, a los problemas de la doctrina de Brentano, cuyo problema central puede ser resumido en solo poder dar cuenta de la distensión del presente de un modo derivado, dado que todo vínculo con el pasado está mediado por 
la asociación originaria que Husserl entiende en términos de fantasía. ${ }^{4}$ Frente a esto, los contenidos aprehendidos por las aprehensiones temporales son modificaciones de la sensación originaria. Con todo, el carácter particular de estos contenidos no queda completamente establecido por la teoría husserliana (Brough 2017, nota 15). A lo cual podemos agregar que, si las aprehensiones de pasado interpretaran fantasmas, sería difícil distinguir entre el recuerdo primario y la rememoración en el sentido corriente.

La teoría del tiempo husserliana sostiene que los recuerdos primarios tienen una naturaleza diferente que la de los recuerdos secundarios (Hua X, p. 46/p. 67). Esto se observa de modo patente en las funciones que ambos actos cumplen. Mientras que el recuerdo secundario supone la percepción y opera una reproducción de algo que ya pasó definitivamente, el recuerdo primario asiste a la percepción a constituirse como acto. En ese sentido, el recuerdo secundario supone el recuerdo primario. En otras palabras, si quiero recordar la melodía que escuché esta mañana, debo haber primero percibido dicha melodía. Como esa melodía estaba constituida gracias a la intervención del recuerdo primario, decimos que el secundario lo supone. En así que puede afirmarse que en el recuerdo primario el pasado "es un hecho que está dado él mismo, «percibido»" (Hua X, p. 36/ p. 58). Lo explica Husserl de este modo:

si llamamos percepción al acto en que todo origen se halla, el acto que constituye originariamente, entonces el recuerdo primario es percepción. Pues sólo en el recuerdo primario vemos lo pasado; sólo en él se constituye el pasado y por cierto no vicaria sino presentativamente ( ). La rememoración, por el contrario ( ) nos brinda una mera evocación que presentifica. (Hua X, p. 41/ p. 63)

Entonces, el recuerdo primario es una conciencia originaria de pasado, en tanto que la rememoración es una conciencia reproductiva del pasado. En ambos casos existe una relación con el presente. Por un lado, el recuerdo primario opera en el presente manteniendo presentes los contenidos recién sidos, brindándolos de modo originario como pasados. Por otro, la rememoración es un acto presente que reproduce el pasado de modo no-originario.

\section{Problemas del esquema}

En el primer parágrafo de las Lecciones Husserl introduce una nota. Anticipa allí una modificación que no se llevará a cabo inmediatamente: la posibilidad de cambiar el modelo de constitución a la hora de aplicarlo a algunas instancias de la conciencia. La nota ya es clásica: "no toda constitución responde al esquema aprehensión-contenido de aprehensión" (Hua X, p. 8, nota 1/ p. 29). En ese caso, la advertencia husserliana se dirige a la constitución de los contenidos. Con todo, también la constitución de los actos ocasiona dificultades.

Entre 1906 y 1907 empiezan a aparecer en los textos sobre el tiempo modificaciones conceptuales. La idea de una instancia supratemporal que fundamente el darse de todo lo temporal está latente en el pensamiento de Husserl. En varios ocasiones ya había afirmado que las percepciones temporales eran ellas mismas también

$4 \quad$ Para mayor detalle sobre este punto ver Kretschel 2017. 
objetos temporales (entre otros: Hua X, p. 228, p. 232, p. 237). Entonces: ¿cómo se constituyen estos objetos temporales inmanentes? Si nos limitáramos al esquema aprehensión-contenido de aprehensión, los objetos temporales inmanentes deberían ser resultado de una aprehensión objetivante y un acto debería intencionarlos. Pero, si postulamos que la constitución de los actos se da a partir de actos de un nivel superior, nos vemos conducidos directamente a un regreso infinito. Requeriríamos de un acto de un nivel superior al segundo que lo constituyera y así sucesivamente. Este sería uno de los motivos del surgimiento de la noción de conciencia absoluta. Es decir, como un modo de resolver el regreso infinito implicado en la constitución de los actos según el esquema.

Con todo, es necesario analizar si la relación entre este problema y la postulación de la conciencia absoluta es tan directa. Como ya señalamos, las críticas al esquema surgen cuando la indagación se orienta hacia la constitución de las unidades inmanentes. Es decir que, mientras se mantenía en la dirección de los objetos trascendentes, la teoría esquemática era válida. Brough señala tres problemas en la aplicación del esquema a la constitución de la conciencia temporal (Brough 1977, pp. 91-92):

1. El primero, señalado como el más importante, radica en la modificación de una de las tesis que sostenían el esquema: la neutralidad de los contenidos. La sensación no es neutral, afirma Husserl hacia 1907, sino que es presente. La sensación es vivida como ahora y no puede ser interpretada ni como pasada, ni como futura. Recordemos que la neutralidad de los contenidos estaba asociada a la posibilidad de que los mismos contenidos puedan ser interpretados de diferente manera, teniendo como referencia trascendente distintos objetos. Un ejemplo de esto son las conocidas figuras de la Gestalt, donde a través de una misma imagen podemos percibir distintas figuras. Esta característica de la sensación no se ve modificada, sino que se cuestiona la neutralidad de los contenidos en relación con el tiempo: "A los contenidos primarios que se extienden en el ahora no les es posible cambiar su función temporal" (Hua X, p. 322), sobre todo teniendo en cuenta que "la sensación es conciencia del ahora" (Hua X, p. 324).

2. El segundo, que coincide con la otra tesis basal del esquema, radica en el carácter arbitrario de la aprehensión de tiempo. Una consecuencia controversial del esquema es que, si la aprehensión constituye la temporalidad de la sensación, el mismo contenido puede ser interpretado como presente, pasado o futuro. Pero, se pregunta Husserl: "¿puede un contenido, en efecto, ser aprehendido en un modo temporalmente distinto y entonces al mismo tiempo, por ejemplo, también ser aprehendido como el mismo contenido idéntico en un modo diferente?" (Hua X, p. 319) Esta pregunta se responde negativamente, considerando la modificación de la primera tesis, dado que antes de dicha modificación los contenidos eran considerados neutrales, ni presentes, ni pasados, ni futuros.

3. El tercero apunta al regreso infinito de la constitución de los actos, indicado más arriba.

Husserl comienza, entonces, a indagar cómo se constituyen las vivencias. Compara la duración de las cosas, los objetos trascedentes, con la duración de las vivencias, 
contenidos y aprehensiones. Plantea que, al igual que los objetos, las vivencias se escorzan. Tenemos, así, continuos de sensaciones y continuos de aprehensiones. Es necesario pensar, entonces, cómo se da la constitución de estos continuos. ¿Cómo se conforman como unidad? Se afirma: "lo que sea que esté dado él mismo como un objeto individual está dado como la unidad de una multiplicidad absoluta que no está dada" (Hua X, p. 284). Las vivencias, entonces, en tanto objetos inmanentes, presuponen una multiplicidad de apariciones a las cuales no se tiene acceso: el flujo de la conciencia se forma e interconecta de un modo único. Se impone, aquí, una distinción dentro de la inmanencia. Mientras que, por un lado, separamos a los objetos trascendentes de las vivencias que los constituyen, por otro, se diferencia en el plano de inmanencia el dominio de las vivencias del flujo absoluto constituyente:

Inmanente puede significar la antítesis de trascendente, y entonces la cosa temporal, el sonido es inmanente; pero también puede significar aquello que existe en el sentido de la conciencia absoluta y entonces el sonido no es inmanente. (Hua X, p. 284)

Es decir que, si nos manejamos con una acepción restringida de inmanencia, inmanente es sólo la conciencia absoluta. Y es fundamental aquello que la distingue tanto de los objetos trascendentes, como de los inmanentes. Estas dos esferas de la realidad se dan de modo temporal "bajo la forma irreductible de las realidades individuales" (Hua X, p. 274). La conciencia absoluta, por el contrario, se posiciona por sobre toda categoría temporal, "más allá de toda objetivación" (Hua X, p. 286). En efecto, en ella se constituye en sentido último toda objetivación. Hay que entenderla como "la forma de la objetivación de algo individual, como la forma de todo posible aparecer" (Hua X, p. 294). Como podemos observar, se establece una equiparación entre el proceso de temporalización y el proceso de objetivación: ser temporal significa devenir objeto. Y, en este sentido, la conciencia del tiempo es no sólo el origen del tiempo, sino también el origen de la experiencia objetiva:

Donde la conciencia no lleva a cabo una síntesis, donde, por ejemplo, ella no pone a través de un acto identificante la identidad de algo que dura en una duración, no hay tampoco ninguna duración, sino sólo un flujo de contenidos capaz de ser caracterizados determinadamente. Esta caracterización tiene lugar en su momento a través de una objetivación de los contenidos como contenidos y a través de los actos que constituyen la objetividad. (Hua X, p. 296, la cursiva es de Husserl)

Ahora bien, las primeras indicaciones respecto a la conciencia absoluta son, con todo, ambiguas respecto a su modo de operar. Se habla de "percepción" de las unidades inmanentes (Hua X, p. 272), lo cual se contrapone a la idea que ya habíamos resaltado de que las vivencias no son percibidas, sino vividas. Y, según eso, las unidades inmanentes deberían constituirse del mismo modo que los objetos trascendentes. Se pone de manifiesto en estas investigaciones una teoría que se va elaborando al enfrentarse a sus propias dificultades. En las palabras de Husserl:

De ninguna manera pretendo ofrecer este análisis como el definitivo: no puede ser nuestra tarea aquí resolver el más difícil de todos los problemas fenomenológicos, el problema del análisis del tiempo. Lo que me importa es sólo correr un poco el velo de este mundo de la conciencia del tiempo, tan rico en misterio que hasta ahora había estado oculto para nosotros. (Hua X, p. 276) 
En formulaciones posteriores la conciencia absoluta constituyente de tiempo soluciona los problemas del esquema a partir de dos condiciones fundamentales. $\mathrm{La}$ primera es que se autoconstituya y, con ello, no sucumba al regreso de la constitución. La conciencia absoluta, entonces, no sólo produce la constitución de los actos, sino también su propia constitución. La segunda condición es que la conciencia como origen de la temporalidad no sea temporal. Está por fuera del tiempo y constituye todo aquello que sí es temporal. Explicaremos en adelante cómo operan estas condiciones.

\section{La conciencia absoluta}

Si bien la idea de la conciencia absoluta ya había aparecido hacia 1907, se producen posteriormente modificaciones fundamentales en la caracterización de esta instancia de la conciencia temporal. Sobre todo, si tenemos en cuenta que las primeras formulaciones en torno a esta noción eran dependientes aún del esquema aprehensióncontenido de aprehensión. Aún cuando Husserl ya había dejado establecida la diferencia de naturaleza entre los aspectos constituidos y los constituyentes de la conciencia, restaba todavía precisar en qué consistía la conciencia absoluta. Siguiendo a Brough, podemos afirmar que a partir de 1909 los análisis sobre la conciencia absoluta tienen un mayor nivel de desarrollo y hacia 1911 la teoría de la instancia última constituyente del tiempo ya está consolidada (Brough 1977, p. 92). Dos cuestiones fundan principalmente esta interpretación. Por una parte, el hecho de que se deja de explicar la conciencia del tiempo con el esquema aprehensión-contenido de aprehensión. Por otra parte, la teoría de la constitución del tiempo se explicita como una teoría del vivenciar (erleben) de los objetos temporales inmanentes. En este sentido, la conciencia absoluta no es una conciencia perceptiva, sino que remite a un último nivel de percatación que tenemos de nuestras vivencias. Junto con estas dos características nota Brough una modificación en la noción de "sensación" (Empfindung). Anteriormente el término refería a un contenido. De hecho, lo hemos usado hasta el momento en esta investigación como sinónimo de contenido. Sin embargo, a partir de 1909, Empfindung mentará el sentir del contenido. Es decir, la vivencia no-intencional no será entendida como el contenido sentido, sino como el sentir de un contenido. Lo que nos retrotrae a una cuestión planteada. La teoría del tiempo que Husserl pretende establecer toma como dato un fenómeno inmanente que es la duración, fenómeno que tiene más que ver con un sentir, que con un contenido en sentido estricto.

Las investigaciones sobre la conciencia absoluta implican cambios conceptuales que se hacen evidentes en modificaciones terminológicas. En el marco del esquema aprehensión-contenido de aprehensión, los actos eran constituidos por aprehensiones temporales presentes, pasadas y futuras. Ahora la conciencia absoluta es la que se encarga de la constitución de las unidades inmanentes. Según esto la distensión temporal del presente no está dada por aprehensiones, sino por las fases de la conciencia absoluta. Si pensamos la conciencia como un flujo continuo que tiene un punto de inicio, a ese punto lo llamamos ahora. Sobre cada ahora la conciencia impone su estructura triple. Lo que antes eran las aprehensiones de tiempo, es en este caso el complejo protoimpresión-retención-protensión. La protoimpresión es la fase de la conciencia que intenciona el punto de inicio, el material nuevo que ingresa a 
la conciencia. A su vez, la retención se dirige hacia lo que ya se dio, mantiene en la presencia los aspectos recién sidos del objeto que estamos percibiendo y da cuenta de la distensión del presente en dirección al pasado. Finalmente, la fase de la conciencia absoluta que constituye el futuro, la protensión, es una conciencia dirigida de modo vacío al porvenir. Aunque Husserl no se dedica especialmente a su descripción en los textos tempranos sobre el tiempo, creemos que es posible al menos sostener que su intencionalidad anticipa que todo lo que se dé a la conciencia en el futuro, se dará de modo temporal. ${ }^{5}$ Como podemos ver, las funciones de la protoimpresión, la retención y la protensión se corresponden con aquellas de la percepción-ahora, el recuerdo primario y la expectativa primaria, respectivamente. Con todo, las nuevas elecciones terminológicas implican transformaciones conceptuales importantes. Las aprehensiones de tiempo eran consideradas, como su nombre lo indica, aprehensiones $\mathrm{y}$, en ese sentido, como instancias constituidas. Las fases de la conciencia, por el contrario, no son constituidas, sino constituyentes. Esto implica, también, que no son temporales:

\section{El flujo de los modos de conciencia no es un proceso; la conciencia del ahora no es ella misma ahora. La retención que existe 'junto' con la conciencia del ahora no es 'ahora', no es simultánea al ahora, y no tendría sentido decir eso. (Hua X, p. 333, la cursiva es de Husserl)}

Es decir que "ahora" remite al objeto, éste se manifiesta como ahora a través de la protoimpresión (modo de conciencia del ahora). Pero la protoimpresión no tiene ella misma un lugar en el tiempo, sino que ella es la condición de posibilidad para que los lugares de tiempo se constituyan; por ella es que podemos hablar de que un contenido está siendo sentido ahora. Lo mismo, respecto a la retención. Ni pasada, ni presente, ni simultánea, las determinaciones temporales son propias de los objetos y no de las fases de la conciencia absoluta, ellas "son no-temporales; esto es: nada en el tiempo inmanente" (Hua X, p. 333-334). No obstante, en ocasiones, Husserl se refiere a la instancia última como "temporalidad pre-inmanente" o "quasi temporal" (Hua X, p. 381), aludiendo a su carácter constitutivo de la temporalidad inmanente y, a la vez, a cierta ordenación que le sería intrínseca. Esta ordenación pre-temporal del flujo sería la condición de posibilidad del orden que atañe a la temporalidad inmanente y no es otra cosa que el derivar unas de otras las sensaciones en retenciones y en modificaciones de estas retenciones de manera continua.

Señalamos que las fases de la conciencia absoluta son constituyentes y no constituidas. Esto habla de la naturaleza especial que caracteriza a la instancia de fundamentación última de la conciencia. Aunque pudiera parecer absurdo -afirma Husserl-el flujo en su dimensión absoluta no sólo constituye los objetos inmanentes, sino también se constituye a sí mismo:

Hay un sólo, un único flujo de conciencia (quizá dentro de una conciencia última) en el cual tanto la unidad del tono en el tiempo inmanente y la unidad del flujo de la conciencia él mismo se constituyen a la vez. Tan chocante (cuando no en un principio también

En los textos tempranos Husserl no caracteriza en profundidad la protensión, sino que se dedica con más detalle a la retención. En los escritos genéticos sobre el tiempo, Die Bernauer Manuskripte über das Zeitbwusstsein, podemos encontrar junto con una revisión integral del funcionamiento de la conciencia temporal descripciones sistemáticas del operar de la protensión. 
absurdo) como puede parecer decir que el flujo de conciencia constituye su propia unidad, es, sin embargo, un hecho que lo hace y eso es algo que puede hacerse inteligible sobre la base de la constitución esencial del flujo. (Hua X, p. 378)

Es posible destacar varias cuestiones en la cita anterior. En primer lugar, algo que ya fue mencionado y es que la conciencia puede ser pensada como un flujo y, enfatiza Husserl, "un sólo, único flujo". Se señala, además, una posibilidad que luego será rechazada: que exista una "conciencia última" que subsuma al flujo. Pero, de hecho, que el flujo se autoconstituya da cuenta ya de su carácter último. Una conciencia más allá del flujo sería una conciencia inconsciente y no es dado para la fenomenología sostener tal afirmación. ${ }^{6}$ En segundo lugar, observamos que tanto los objetos inmanentes como el propio flujo son constituidos por el mismo flujo y esto "puede hacerse inteligible sobre la base de la constitución esencial del flujo". La metáfora del flujo apunta a entender la conciencia como un fluir que, en su estrato más originario y pre-objetivo, es un fluir de protoimpresiones que derivan en retenciones que, a su vez, se modifican continuamente en retenciones de retenciones.

Ahora bien, las retenciones poseen una doble intencionalidad (Hua X, p. 379). Por una parte, apuntan a la constitución de los objetos, junto con las protoimpresiones y las protenciones. Por otra parte, se dirigen al propio flujo, en la medida en que se modifican a sí mismas de modo continuo. Estas dos direcciones de la retención son: la intencionalidad transversal (Querintentionalität) y la intencionalidad longitudinal (Längsintentionalität), respectivamente. Respecto a la primera, resulta de interés tener en cuenta que el término empleado en castellano pierde un poco el sentido del mismo en alemán. Para profundizar, entonces, en el sentido del término habría que considerar que Querintentionalität remite a Querschnitt, que es el término alemán que se traduce aquí como fase, en el sentido específico de corte. Es decir que cada fase, cada corte discreto en el flujo de la conciencia es un Querschnitt. Sobre él opera la intencionalidad transversal de la conciencia, posibilitando la conformación de los objetos, en la distensión del presente.

La intencionalidad longitudinal de la retención es, por su parte, la encargada de la constitución del flujo. Y se habla, entonces, de auto-constitución, dado que las retenciones conforman el flujo y, en ese mismo hecho, lo constituyen:

Creemos, entonces, que la unidad del propio flujo se constituye en el flujo de la conciencia como un orden uni-dimensional y quasi- temporal a través de la continuidad de las modificaciones reproductivas y a través de las circunstancias que esas modificaciones son, continuamente, reproducciones unas de otras, de reproducciones que continuamente las han precedido. (Hua X, p. 380)

El continuo modificarse de las retenciones apunta a la autoconstitución del flujo. Hay que tener en cuenta, no obstante, que, aunque con fines descriptivos sea conveniente distinguir entre ambas intencionalidades de la retención, ambas operan siempre juntas, como dos caras del mismo proceso.

Ahora bien, otra transformación que supone el abandono del esquema para la instancia absoluta tiene que ver con el otro aspecto del esquema: los contenidos. Las aprehensiones temporales interpretaban contenidos y así se constituía el acto y la

6 Husserl desarrollo este argumento en el apéndice IX de las Lecciones. Al respecto se puede leer la crítica de Derrida en La voz y el fenómeno. 
referencia objetiva. Mientras que la aprehensión actual apuntaba a la sensación, no quedaba claro cuál era el contenido respecto a las aprehensiones pasadas y futuras, ya nos hemos referido a los problemas implicados en la noción de fantasma. En la nueva concepción de la instancia última de constitución, sólo la protoimpresión está en relación con la sensación, dado que la sensación es ahora, siempre presente. La fase retencional se concibe, entonces, como una modificación de la fase actual. Es decir, no implica una nueva interpretación de una sensación, sino que es la conservación modificada de la protoimpresión en sí misma.

El surgimiento de la conciencia absoluta da cuenta de una transformación respecto al modelo de constitución. Toda una discusión se centra en qué status adquiere dicha transformación. La primera recepción de la teoría del tiempo husserliana en la que se suele agrupar a Rudolf Boehm, Robert Sokolowski, Rudolf Bernet y John Brough ha coincidido en aceptar que Husserl lleva a cabo un abandono del esquema aprehensión-contenido de aprehensión a partir de los estudios tempranos sobre el tiempo (Mensch 2010, p. 153). Esta primera posición respecto al modelo de constitución fue restringida en el caso de Brough. Y, a partir, de la constatación de Dieter Lohmar del empleo del esquema en textos posteriores, incluso hasta finales de los años '1930 (Lohmar 2006, p. 406), James Mensch se encargó de fundamentar la vigencia del esquema para la constitución de objetos durante toda la obra de Husserl. Así, sostiene: "afirmar que los fenómenos constituyentes de tiempo no son ellos mismos temporales es también afirmar que este esquema no se aplica a ellos" (Mensch 2010, p. 160). En este sentido, creemos válido hablar de abandono respecto a los elementos constituyentes y seguir sosteniendo el esquema en relación con la constitución de la objetividad.

\section{Los niveles de la temporalidad}

Ya las primeras indicaciones respecto al carácter absoluto de la conciencia determinaron una distinción en el terreno de la inmanencia: el dominio de las vivencias debía ser distinguido del dominio del flujo no objetivable. Si sumamos a estas el ámbito de la realidad empírica trascendente, es posible distinguir tres niveles de la existencia, que se expresan en tres niveles de la temporalidad. Husserl refiere en varias oportunidades a esta distinción. La más conocida es, quizá, la que aparece en el $\S 34$ de las Lecciones. Según indica Edith Stein en una nota, este parágrafo fue conformado a partir del texto que encontramos como $\mathrm{N}^{\circ} 40$ "Niveles de la objetividad" en Husserliana X (Hua X, 287, nota del editor). Lo curioso es que en el texto inspirador del famoso parágrafo de las Lecciones los niveles de la conciencia del tiempo se encuentran ordenados exactamente al revés. Las modificaciones introducidas por Stein en este pasaje, creemos, apuntan a hacer más comprensible el desarrollo de las Lecciones. Una estratificación de los niveles de constitución temporal permite ordenar la exposición de los parágrafos siguientes, en los cuales se enfrentan las características de la conciencia absoluta con las de las unidades constituidas por ella. Con todo, y según indica su título, el texto original busca distinguir "niveles de la objetividad". Lo cual es plenamente evidente en el nivel 3: el ser empírico, real y constituido. Mientras que el nivel 1 puede leerse tanto como un dominio de la realidad, como de la instancia constituyente del tiempo; el nivel 2 apunta sólo a una dimensión de la temporalidad. Entonces: ¿por qué 
niveles de la objetividad e introducir en ellos un nivel puramente temporal? ¿Es la existencia de las vivencias meramente una dimensión de la conciencia constituyente del tiempo? En principio, lo que podemos garantizar es una relación muy estrecha entre objetivación y temporalización. Ya nos hemos referido a este tema más arriba, en el momento en que caracterizábamos la conciencia absoluta constituyente del tiempo como el proceso de objetivación. Sin síntesis, sin duración, no hay objeto. El tiempo es la forma que hace posible la aparición -es decir: la existencia- de los objetos trascendentes. Pero: ¿cuál es el status de los objetos inmanentes? ¿Son un mero "puente" hacia la trascendencia?

En los últimos años ha surgido una discusión muy intensa acerca de la distinción en tres niveles de la conciencia temporal, asumida hasta ese entonces la necesidad de tal distinción. Esta interpretación, llamada standard o Brough/Sokolowski, fue cuestionada por primera vez en 1999 por Dan Zahavi en su libro Self-Awareness and Alterity. En el marco de un estudio de la auto-conciencia, Zahavi consideró la exigencia de que el nivel de los actos fuera independiente. Posteriormente la discusión fue retomada por numerosos académicos (Zahavi 2011, p.14). Finalmente, en 2011, a partir de una intervención de Neal de Roo, Brough y Zahavi parecen haber establecido cuál es su última palabra sobre el debate. Retomemos, entonces, brevemente en qué consiste tal debate.

\subsection{Las críticas de Zahavi a la "interpretación standard"}

En su obra de 1999 Zahavi investiga el tema de la auto-conciencia (Self-Awareness) y toma partido en contra de una concepción reflexiva de la auto-conciencia. Las teorías de la reflexión, tal como las describe, conducen inevitablemente a regresos infinitos. En ese sentido señala que la autoconciencia debe ser inmediata, tácita y no temática (Zahavi 1999, p. 27). Alude, a su vez, a la relación intrínseca que existe entre la autoconciencia y la temporalidad y señala la necesidad de combinar una teoría plausible de la autoconciencia con una explicación sobre la temporalidad, dado que: "después de todo, no soy sólo consciente de mi percepción actual, sino también capaz de recordar mi experiencia pasada como mía" (Zahavi 1999, p. 36, la cursiva es de Zahavi). Según esto, piensa que en la fenomenología del tiempo de Husserl es posible encontrar una descripción de la temporalidad que dé cuenta de modo ajustado de la autoconciencia. Si bien su objetivo primario no es un estudio del tiempo, con todo, como él mismo afirma, sus estudios pueden conducir a esclarecer ciertos problemas de la teoría del tiempo husserliana (Zahavi 1999, p. 69), En especial, se concentra en los análisis sobre la dimensión última de constitución.

Zahavi se posiciona frente a la interpretación standard que resume de la siguiente manera. Si distinguimos respecto a los objetos constituidos una dimensión constituyente que los hace aparecer, debemos distinguir en relación con los actos -constituidos- una dimensión que permita su manifestación. Mientras que para la dimensión trascendente tenemos a los actos como lo que posibilita su darse, la conciencia absoluta es la que da cuenta del aparecer de los actos. El punto es que, si la conciencia absoluta lo que hace es dar a los actos como objetos -es decir que la manifestación primigenia es objetivante-, nos enfrentamos, entonces, a una teoría reflexiva de la autoconciencia que conlleva un regreso infinito.

Sin embargo, lo que cabría preguntarse, sostiene Zahavi, es si realmente la conciencia interna es autoconsciente. Por un lado, si no lo es, no se explica de dónde proviene la autoconciencia de los actos. Por otro lado, si respondemos 
afirmativamente, debemos explicar cómo se da tal autoconciencia: si se da del mismo modo en que se dan los actos, tenemos nuevamente un regreso infinito, porque requeriríamos de un acto de grado superior que explicara la autoconciencia del acto que hace autoconsciente al primer acto y así sucesivamente; pero, si, por el contrario, estamos dispuestos a afirmar que hay una autoconciencia implícita en la conciencia interna, no se explica por qué no pueden ser los mismos actos conscientes de sí. Esto es, no se justifica la necesidad de la conciencia absoluta. En efecto, para Zahavi, la postulación de la conciencia absoluta como una dimensión separada de los actos representa una multiplicación innecesaria de las dimensiones de la conciencia. Sería más simple pensar que la conciencia interna es "solo" la conciencia interna de cada acto: ese saber prereflexivo acerca de que tal o cual acto es un acto mío. Por tanto, la conciencia interna debe ser la autoconciencia prereflexiva del acto. De aquí se sigue la afirmación de Zahavi según la cual "las investigaciones husserlianas sobre la conciencia interna del tiempo no son otra cosa que una investigación sobre la temporalidad de la autoconciencia prereflexiva" (Zahavi 1999, p. 71). Como se desprende de esto, efectúa en su argumentación un desplazamiento de la cuestión de la autoconciencia al tema de la temporalidad, para terminar equiparando ambas investigaciones.

En esta lectura de la conciencia interna como la conciencia prereflexiva del acto se funda la discusión acerca de la división en niveles de la conciencia. La tesis de Zahavi parece conducir a la eliminación del segundo nivel; es decir: el nivel del tiempo inmanente. Los actos sólo se constituyen como objetos inmanentes en un segundo momento, a través de una mirada reflexiva sobre el curso de conciencia. Las vivencias fluyen unas en otras y tenemos de ellas una conciencia prereflexiva que permite que las identifiquemos como propias. Si bien esta interpretación parece simplificar la teoría del tiempo husserliana y, a la vez, dar cuenta de una teoría de la autoconciencia no objetivante, resulta difícil de ser sostenida textualmente. Como vimos, el propio Husserl se refiere a las vivencias como objetos inmanentes, mentando con esto no sólo las percepciones, sino también los contenidos. De hecho, en los textos husserlianos el segundo nivel de la conciencia parece, a veces, estar constituido por dos flujos: un flujo de actos y otro flujo de contenidos, ambos con cierto carácter objetivo.

Con todo, es necesario advertir que, si bien Zahavi se opone a la necesidad de una distinción de las dimensiones inmanentes de la conciencia, lo que parece negar en un principio toda posibilidad de diferenciar las vivencias del curso absoluto, en un texto de 2005 explicita su punto de vista y afirma que:

Cuando Husserl asegura que la vivencia es constituida en la conciencia interna del tiempo, no está diciendo que la vivencia es traída a la donación por otra parte de la subjetividad, como si una parte tomara a la otra como su objeto. Más bien, decir que una vivencia es constituida en la conciencia interna del tiempo es decir que es traída a la conciencia por sí misma. Es llamada conciencia interna del tiempo porque ella pertenece intrínsecamente a la estructura íntima de la propia vivencia. (Zahavi 2005, p. 65, la cursiva es de Zahavi)

Zahavi sostiene aquí la idea de que la conciencia interna del tiempo es la autoconciencia de la vivencia. No aceptar eso implicaría que las vivencias sean tomadas como objetos por el flujo absoluto. Lo cual, como hemos visto, redundaría en una teoría reflexiva de la autoconciencia y, con ello, en un regreso infinito. Para 
fundamentar su punto, pone el énfasis en el carácter interno que se le asigna a la conciencia del tiempo. “¿Interno a qué?”, cabría preguntar. Justamente, a la vivencia.

Con todo, más adelante en el texto agrega:

Insistir en que el propio flujo de la conciencia del tiempo es la donación prereflexiva de las vivencias no es denegar la distinción entre nuestras vivencias singulares y transitorias y la dimensión perdurable del vivenciar, esto es, entre die Erlebnisse und das Erleben... (Zahavi 1999, p. 66, la cursiva es del autor)

Acepta aquí, entonces, una diferenciación que es practicable en la inmanencia de la conciencia entre nuestras vivencias y el flujo. En efecto, no son lo mismo las vivencias intencionales y objetivantes que la donación prereflexiva del flujo; podemos referirnos a vivencias de variado tipo, en tanto que la autoconciencia que tenemos de ellas es siempre la misma. Con todo, esta posibilidad de diferenciar las vivencias del propio vivenciar no implica de ninguna manera la capacidad de separar la inmanencia en dos dominios o realidades. Hacerlo supondría aceptar que existe un ámbito de donación preobjetiva sobre el que se montan las vivencias, cuando la vida de la conciencia es, de hecho, inseparable de sus contenidos.

En un último artículo sobre el tema Zahavi confirma las tesis recién expuestas (la distinción y la no-separabilidad entre die Erlebnisse y das Erleben) y afirma que la discusión con Brough no tiene tanto que ver con la cantidad de niveles que tiene la conciencia temporal, sino que debe concentrarse en las siguientes dos cuestiones (Zahavi 2011, pp. 21-.22):

1. La tesis de que las vivencias se dan prereflexivamente como objetos inmanentes.

2. La necesidad de que más allá de la autoconciencia prereflexiva de los actos haya una autoaparición adicional y distinta del flujo.

En relación con 1., hay que destacar que referirse a las vivencias como objetos inmanentes tiene una amplia apoyatura textual. Con todo, no sería la noción de objeto la más conveniente para referirse a las vivencias, dado que implicaría una función objetivante de la conciencia absoluta, característica que el propio Husserl se encarga de criticar. La cuestión puede salvarse si se toma la noción de objeto en sentido amplio. En este sentido, Brough se ha referido a las vivencias como unidades inmanentes, tesis que Zahavi, dice, podría aceptar.

Respecto a 2., las posibilidades de un acuerdo parecen más difíciles. Zahavi observa en los textos de Brough dudas en relación con la existencia de un flujo "independiente". El problema central, y volveremos sobre esto más abajo, tiene que ver con la posibilidad de dar cuenta de la unidad de la experiencia si no existe un flujo total en el que se integren todas las vivencias. Podemos anticipar que, para Zahavi, la unidad del flujo está garantizada por la certeza que tengo de que una vivencia es mía. No habría necesidad de otra instancia para dar cuenta de esa pertenencia. Con todo: ¿no tengo, más allá del saber acerca de que una vivencia es mía, un conocimiento no-temático acerca del antes y el después de una vivencia? ¿de si una vivencia es actual o pasada respecto a otra? Y ¿pueden las vivencias por sí solas darse ese "conocimiento"? 


\subsection{Brough y el problema de las vivencias}

En un artículo de 2010 Brough afirma que: "La fenomenología de la conciencia del tiempo de Husserl es un esfuerzo por entender la vida de la conciencia en su identidad con la conciencia temporal" (Brough 2010, p. 21). Al tomar los estudios husserlianos en su conjunto -es decir: más allá del período de las Lecciones y los textos complementarios-, se observa que la teoría sobre el tiempo excede el problema del darse del objeto temporal. La conciencia temporal opera como la forma sobre la cual se organiza la vida de la conciencia en su conjunto. Aquello que distingue a la conciencia temporal de las otras dimensiones de la constitución es el hecho de que se autoconstituye. La autoconstitución de la conciencia coincide, a su vez, con su automanifestación. A diferencia de los objetos trascedentes y de las vivencias, la conciencia absoluta no requiere de una instancia por fuera de sí para manifestarse, ella se da a sí misma. En este sentido, puede decirse que es absoluta, dado que no depende de ningún otro aspecto de la conciencia. Esta suerte de independencia de la conciencia no debe ser entendida como algo separado de las vivencias. La inmanencia de la conciencia conforma un solo dominio en el cual, como sostiene Zahavi, pueden hacerse distinciones que habría que considerar distinciones de razón. Ahora bien, la posición de Brough se amplía en dirección a los textos posteriores sobre el tiempo y considera el problema de la temporalidad en un sentido más amplio. Con todo, si bien su artículo "The Emergence..." apareció tempranamente respecto a lo que podríamos llamar la historia de la recepción de la fenomenología del tiempo husserliana, los cambios operados en su interpretación no han sido muy radicales. Si nos ceñimos a los distintos períodos de los textos, la interpretación de Brough seguiría estando vigente dentro del marco de los escritos que se recogen en Husserliana $X$.

En el artículo que nombramos al comienzo de esta sección Brough enfrenta las objeciones formuladas por Zahavi. En primer lugar busca sostener la necesidad de un flujo de vivencias conformadas, al menos, como unidades preobjetivas. La idea central es que: "nuestra conciencia prereflexiva no se nos presenta como si fuera un puré (porridge) homogéneo en el cual todo momento es igual a otro" (Brough 2010, p. 38). Es decir, no vivimos los actos fusionados entre sí, sino distinguidos; los actos se dan como unidades discretas. En este sentido puede afirmarse que el flujo de la conciencia posee un relieve, diferenciaciones, vivencias que se destacan sobre otras. El ejemplo empleado es el del mar y las olas, ejemplo traído de Husserl y utilizado anteriormente por Zahavi (Brough 2010, p. 39). ${ }^{7}$ Según esta representación de la vida de la conciencia, el flujo absoluto sería el mar, en el cual podemos identificar las olas como unidades discretas, pero, a la vez, dependientes del flujo. A su vez, la imagen permite mostrar que el flujo no está separado de las vivencias.

En segundo lugar, la visión de la dimensión de las vivencias en términos de relieve es complementada con una crítica por la negativa. Se objeta la posibilidad de que el segundo nivel de la conciencia temporal advenga sólo reflexivamente y se plantea el siguiente problema: ¿cómo la reflexión constituiría los actos como objetos si estos no tuvieran previamente cierta unidad que permitiera distinguirlos uno de los otros? Es decir, si el flujo de la conciencia fuera un flujo indiferenciado, sin

Brough refiere a la metáfora tomada por Zahavi (1999, p. 77) del manuscrito L I 15 2b, pero también a otra aparición de una imagen similar en Hua Mat VIII, 362-363. 
distinciones, sin nada que se destacara: ¿cómo se dirigiría la reflexión a esta o a aquella vivencia? Así, la necesidad de un segundo nivel pre-objetivo en la conciencia temporal parece establecido. Es importante recordar aquí que si bien los primeros enfoques de Zahavi parecían dejar de lado la posibilidad de las distinciones entre las vivencias, posteriormente asume la misma posición que Brough en este respecto.

Sin embargo, las críticas de Brough no se detienen aquí, sino que aborda la posibilidad de que la conciencia absoluta pueda identificarse sin más con la conciencia prereflexiva de los actos. Primero, plantea dos problemas. La tesis central en esta parte es que si la conciencia interna fuera intrínseca a los actos, éstos debieran autoconstituirse. Lo que va a probar es que, como los actos no pueden autoconstituirse, ni ser constituidos por otros actos, entonces es necesario que la conciencia absoluta sea distinguible de ellos. Así, en el primer problema identificamos la siguiente cadena de argumentos:

1. Si la conciencia interna fuera la conciencia prereflexiva de los actos, y esta coincidiera con la dimensión constituyente de la conciencia, entonces los actos se constituirían a sí mismos.

2. Si los actos se constituyeran a sí mismos, y teniendo en cuenta la estructura retención-protoimpresión-protensión, los actos retendrían actos. Se entiende que si la conciencia absoluta es la conciencia de los actos, entonces las retenciones son función de los actos.

3. Pero, como los actos son objetivantes y ellos tienen que dar cuenta de las retenciones, entonces: ¿las retenciones (y las protensiones) serían objetivantes?

4. Si esto fuera así, no podríamos hablar de una conciencia prereflexiva de los actos.

Este razonamiento ofrece motivos suficientes para rechazar la interpretación de Zahavi, al menos en su versión más fuerte. Si seguimos a Brough aquí, la lectura alternativa sobre los niveles resulta ser autocontradictoria.

El segundo problema que aborda en este primer momento, puede reformularse en la siguiente pregunta: ¿cómo da cuenta un acto de algo que perdura como lo es mi autoconciencia? Es decir: ¿la autoconciencia de los actos puede brindar autoconciencia del flujo como un todo? La interpretación standard sostiene que no, que no es pensable en qué sentido un acto -o varios unidos no se sabría bien por qué mecanismo- podrían ofrecer una experiencia de la manifestación que perdure de manera unificada. Tanto el primero como el segundo problema, llevan a Brough a concluir que los actos no pueden autoconstituirse, o, más bien, que no pueden efectuar las operaciones que se le atribuyen a la conciencia absoluta. Nos parece interesante destacar aquí la articulación entre estos dos problemas en la estrategia de Brough. Porque si nosotros asumimos, como nos conduce él con bastante gracia a hacerlo, que los actos son unidades discretas, luego es bastante improbable aceptar que estas mismas unidades discretas puedan dar cuenta del flujo como unidad. En ese sentido, la interpretación de Zahavi requería que los actos fueran objetos reflexivamente, porque eso le permitía también pensar el flujo como algo total. Las posteriores modificaciones - o especificaciones-en su teoría, si bien la hacen más fiel a la letra de Husserl, le sacan consistencia. A su vez, creemos que el intento de Zahavi de dar cuenta de una manera consistente del fenómeno de la automanifestación lo 
conduce a descuidar el aspecto constituyente de la unidad de la conciencia temporal. Si bien la simpleza de su propuesta es atractiva, no respeta toda la complejidad que reúne el fenómeno.

Ahora bien, en último lugar, Brough introduce el punto donde la distancia entre su pensamiento y el de Zahavi parece extenderse. La idea de que el flujo absoluto es separado e independiente de las vivencias no la sostienen ni Brough, ni Zahavi, ni, sobre todo, Husserl. En palabras de Brough: "el flujo es consciente de sí sólo en el proceso de ser consciente de las unidades inmanentes" (Brough 2010, p. 43). La pregunta clave no es, luego, la de la separabilidad del flujo, sino si el flujo es algo más que las vivencias. Para Zahavi, la unión entre los actos está dada por el simple hecho de que yo pueda afirmar que son míos y están unidos porque todos me pertenecen. Para Brough, eso es insuficiente. La unidad no es sólo la pertenencia al flujo de vivencias, sino el lugar dentro de la corriente. La sucesión y la coexistencia son notas que le pertenecen a la conciencia prereflexiva intrínsecamente y así opera el flujo absoluto. En este sentido, el flujo absoluto debe ser considerado algo más que las vivencias.

\section{Conclusión}

La fenomenología del tiempo constituye un dominio de investigación tan amplio como complejo. Los esfuerzos de Husserl por esclarecer el carácter de la conciencia del tiempo, sus distintas dimensiones y las distintas experiencias temporales posibles dieron lugar a una profusa obra sobre la cuestión. La primera parte de la misma que comienza con la publicación en 1928 de las Lecciones de fenomenología de la conciencia inmanente del tiempo es con seguridad la más difundida, pero, con todo, raramente abordada como un todo. Nuestra convicción es que tomar la fenomenología del tiempo con una descripción en proceso, permite sopesar las discrepancias que pueden resultar de la teoría. Con esto en mente, nos propusimos aquí llevar a cabo un recorrido por estos escritos, buscando esclarecer los puntos centrales de una teoría temprana del tiempo pasible de ser confrontada con las discusiones más relevantes del estado de la cuestión. En este sentido, decidimos abordar la problemática del modelo de constitución de la conciencia temporal y el problema de las dimensiones, temas que, claro está, no pueden ser separados entre sí por completo. La restricción del modelo aprehensión-contenido de aprehensión a los objetos y la consecuente separación del flujo de conciencia de este modo de constitución da la pauta de una forma del ser y del aparecer que no es objetiva. Junto con esto, la posibilidad de pensar una instancia constituyente separable de lo objetivable parece necesariamente indispensable.

\section{Referencias bibliográficas}

Abella, M. (2005): “Edmund Husserl: Génesis y estructura de las «Lecciones de fenomenología de la conciencia interna del tiempo»", Daímon. Revista de Filosofia, 34, pp. 143-152.

Brough, J. B. (1977): “The Emergence of an Absolute Consciousness in Husserl's Early Writings on Time Consciousness" en Elliston, F. A. y Mc Cormick, P. (Eds.), Husserl. Expositions and Appraisals, Notre Dame/Londres, University of Notre Dame Press. 
Brough, J. B. (1991): “Translator's Introduction”, en Husserl, E., On the Phenomenology of the Consciousness of Internal Time (1893-1917), Dordrecht, Kluwer.

Brough, J. B. (2010): "Notes on the Absolute Time-Constituting Flow", en Lohmar, D. y Yamaguchi, I. (Eds.), On Time-New Contributions to the Husserlian Phenomenology of Time, Dordrecht, Springer, pp. 21-49.

De Roo, N. (2011): "Revisiting the Zahavi-Brough/Sokolowski Debate", Husserl Studies, 27, pp. 1-12.

Husserl, E. (1985): Logische Untersuchungen. Erster Teil. Prolegomena zur reinen Logik. Text der 1. und der 2. Auflage. Husserliana XVIII, La Haya, Martinus Nijhoff.

Husserl, E. (1984): Logische Untersuchungen. Zweiter Teil. Untersuchungen zur Phänomenologie und Theorie der Erkenntnis. In zwei Bänden. Husserliana XIX, La Haya, Martinus Nijhoff.

Husserl, E. (1976): Investigaciones Lógicas, Madrid, Revista de Occidente.

Husserl, E. (1969): Zur Phänomenologie des inneren Zeitbewusstesens (1893-1917), Husserliana X, Tübingem, Max Niemayer.

Husserl, E. (2002): Lecciones de fenomenología de la conciencia interna del tiempo, Madrid, Trotta.

Ingarden, R. (1962): "Edith Stein on her Activity as an Assistant of Edmund Husserl", Philosophy and Phenomenological Research, 23, pp.155-175.

Kretschel, V. (2017): "Hacia una fenomenología del tiempo. Una interpretación de las críticas de Husserl a Brentano en las Lecciones de fenomenología de la conciencia interna del tiempo", Endoxa, 39, pp. 185-201.

Lohmar, D. (2010): "On the Constitution of the Time of the World" en D. Lohmar-I. Yamaguchi (Eds.), On Time-New Contributions to the Husserlian Phenomenology of Time, Dordrecht, Springer, pp. 115-136.

Lohmar, D. (2006): "Synthesis in Husserls Phänomenologie. Das Grundlegende Modell von Auffassung und auffgefasstem Inhalt in Wahrnehmung, Erkennen und Zeitkonstitution", en Fonfara, D., Metaphysik als Wissenschaft, Festchrifft für Klaus Düssing zum 65. Geburstag, Freiburg/München, Verlag Karl Alber, pp. 38-39.

Mensch, J. (2010): "Retention and the Schema", en D. Lohmar-I. Yamaguchi (Eds.), On TimeNew Contributions to the Husserlian Phenomenology of Time, Dordrecht, Springer, pp. 154-168.

Zahavi, D. (2011): "Objects and Levels: Reflections on the Relation Between TimeConsciousness and Self-Consiousness", Husserl Studies, 27, pp. 11-35.

Zahavi, D. (1999): Self-awareness and Alterity. A Phenomenological Investigation, Evanston, Northwestern University Press.

Zahavi, D. (2005): Subjectivity and Selfhood, Massachusetts, MIT. 